\title{
Superior Thermal Dissipation in Graphene Electronic Device through Novel Heat Path by Electron-Phonon Coupling
}

\author{
Ying Zhang, ${ }^{1, \uparrow}$ Yaping Yan, ${ }^{1, \uparrow}$ Jie Guo, ${ }^{1}$ Tingyu Lu, ${ }^{1,2}$ Jun Liu, ${ }^{2}$ Jun Zhou ${ }^{1}$ and Xiangfan $\mathrm{Xu}^{1,3, *}$
}

\begin{abstract}
A bstract
Interfacial thermal resistance (ITR) plays an important role in thermal dissipation across different materials and it has been widely investigated in recent years. In this work, we measured the relative change of the ITR between metal and aluminum oxide treated with $\mathrm{O}_{2}$-plasma. Significant reduction of ITR is observed. The measured data shows that plasma treatment induces an order of magnitude decrease of ITR, which is mainly attributed to the direct electron-phonon coupling across the interface. Scanning thermal microscopy technique measurement of graphene electronic devices on aluminum oxide gave direct evidence for heat dissipation applications by tuning the surface charge carries concentration.
\end{abstract}

Keywords: thermal dissipation; electron-phonon coupling; graphene; metal-nonmetal interface; interfacial thermal resistance Received: 30 September 2019; Accepted: 3 December 2019

Article type: Research article

\section{Introduction}

In graphene-based electronic devices, the Joule heat is generated in graphene and accumulates between the dielectric layer and graphene film during operations. ${ }^{[1]}$ Therefore, the interfacial thermal resistance (ITR) at solid-solid interface, especially metal-nonmetal interface, has aroused extensive attention due to its extremely important role in heat dissipation. ${ }^{[2,3,4,5,6]}$ In general, the thermal energy in metal can be transferred to nonmetal via three channels which can be equivalently derives as a series-parallel thermal network (Fig. 1(a)). ${ }^{[7]}$ Firstly, the upper and lower limits of interfacial thermal conductance (ITC), which is the inverse of ITR, through phonon-phonon interaction across the interface $\left(Q_{3}\right.$ in Fig. 1) are given by acoustic mismatch model ${ }^{[8]}$ and diffuse mismatch model, ${ }^{[5]}$ respectively. Secondly, the electrons transfer thermal energy to the phonons within the metal and then the phonons transfer the energy to the nonmetal side $\left(\mathrm{Q}_{2}\right.$

Center for Phononics and Thermal Energy Science, China-EU Joint Center for Nanophononics, School of Physics Science and Engineering, Tongji University, 200092 Shanghai, China

${ }^{2}$ Department of Mechanical and Aerospace Engineering, North Carolina State University, Raleigh, North Carolina 27695, USA

${ }^{3}$ Zhejiang Province Key Laboratory of Quantum Technology and Device, Zhejiang University, Hangzhou 310027, China

*E-mail:xuxiangfan@tongji.edu.cn (X.Xu)

† These authors contributed equally in Fig. 1). Such a channel acts in series with the first channel, which will further increase the value of ITC ${ }^{[9]}$ While compared with the measured values across a variety of metal-nonmetal interfaces, such as $\mathrm{Pb} / \mathrm{Ti} / \mathrm{Al} / \mathrm{Au}$ (metal) and diamond/ sapphire/ $\mathrm{BaF}_{2}$ (nonmetal) interfaces, large underestimations were observed in models considering the above two channels. Thus it's reasonable to take into account the third channel, namely, the direct coupling between electrons from the metal side and phonons from the nonmetal side $\left(Q_{1}\right.$ in Fig. 1$) .{ }^{[10,11,12]}$

The direct electron-phonon coupling mentioned above has not been experimentally verified yet. Because it is hard to distinguish the direct electron-phonon coupling contribution from other two channels. It has been reported that the ITC across gallium-He II interface at low temperature using the AC technique. ${ }^{[13]}$ Rotkin et al. ${ }^{[14]}$ demonstrated the dominant role of electron-remote interfacial phonon (RIP) coupling in the heat dissipation across carbon nanotube (CNT)- $\mathrm{SiO}_{2}$ interface, which was later proved experimentally by Baloch et al. ${ }^{[15]}$ Nevertheless, unlike those in $\mathrm{CNT}-\mathrm{SiO}_{2}$ interfaces, the upper limit of the contribution of RIP scattering in ITC across unbiased graphene- $\mathrm{SiO}_{2}$ interfaces was found to be $<2 \%$ in voltage-modulated thermal reflectance (VMTR) experiments, ${ }^{[16]}$ letting alone the effect of better conformity of the interface. Therefore, whether the role of direct electron-phonon 


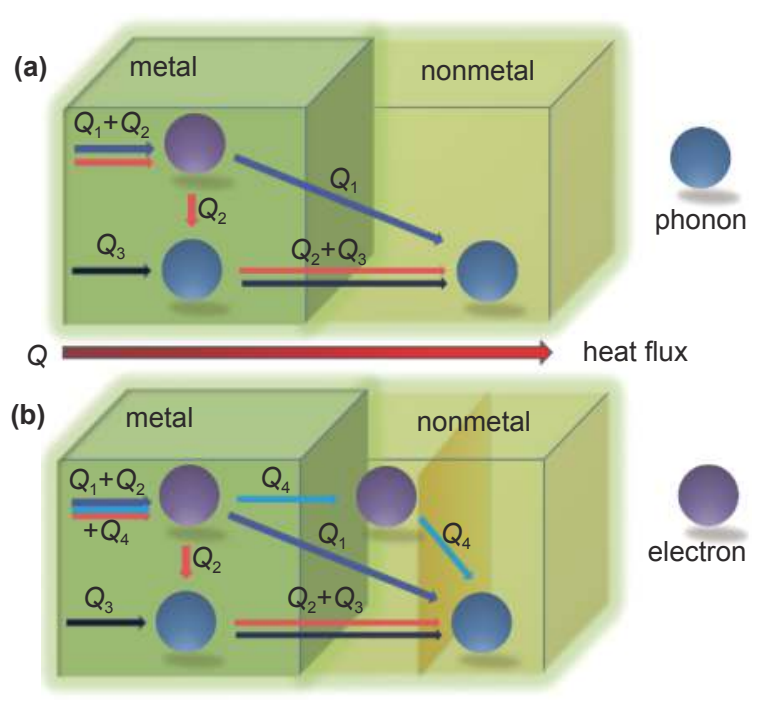

Fig. 1 Schematic illustration of heat conduction from metal to nonmetal across the interface. $Q$ is the total heat current. (a) shows traditional heat path between metal and nonmetal interface and opening a new heat path through electron-phonon coupling at the interface (b).

coupling is important and what the mechanism of direct electron-phonon coupling is across the interface are still under debates. The experimental observation of metal-nonmetal interfacial thermal transport behavior will give us the opportunity to investigate this process.

Here we adopted differential $3 \omega$ method to measure the variations in ITR across $\mathrm{Au}-\mathrm{Al}_{2} \mathrm{O}_{3}$ interfaces after treating the $\mathrm{Al}_{2} \mathrm{O}_{3}$ surface with $\mathrm{O}_{2}$-plasma. Comparing the ITR with and without applying the $\mathrm{O}_{2}$-plasma, we found that the longer treated time of $\mathrm{O}_{2}$-plasma corresponded to smaller ITR. The surface roughness of $\mathrm{Al}_{2} \mathrm{O}_{3}$ was morphologically tested using atomic force microscope (AFM) to eliminate the effect from surface contamination/roughness. Then we deduced the carrier concentration of $\mathrm{Al}_{2} \mathrm{O}_{3}$ under different $\mathrm{O}_{2}$-plasma treatment time by transfer a single layer graphene and measuring its Hall resistance. These results show that with the increase in treatment time of plasma, the carrier concentration is increased. According to the surface states electron-phonon coupling model proposed by $\mathrm{Lu}$ et al. ${ }^{[17]}$ the increase of the charge carrier concentration leads to an increase of Fermi level, which leads to a large increase of ITC. In addition, the results of scanning thermal microscopy technique (SThM) measurement based on grahene $/ \mathrm{Al}_{2} \mathrm{O}_{3}$ devices show a broader space for the application of thermal dissipation.

\section{Experiments}

\subsection{Doping the Interface Between Metal and Nonmetal}

The sample fabrication process is summarized in Fig. 2(a). Nearly 200 atomic layer deposition (ALD) cycles were firstly achieved to deposit $30 \mathrm{~nm} \mathrm{Al} \mathrm{O}_{3}$ in this work. The $30 \mathrm{~nm}$ thickness $\mathrm{Al}_{2} \mathrm{O}_{3}$ layer was deposited onto silicon dioxide substrate under the temperature of $100{ }^{\circ} \mathrm{C}$ by an ALD system in which $\mathrm{AlMe}_{3}$ and $\mathrm{H}_{2} \mathrm{O}$ were used as the precursors of $\mathrm{Al}$ and $\mathrm{O}$, respectively. Subsequently, the samples were treated by $\mathrm{O}_{2}$-plasma with different times varying from four minutes to ten minutes (marked as S4, S5, S6, S7, S8, S9, S10) (Table 1.) and one sample without any treatment (marked as S0) was regarded as reference. The plasma cleaner machine was used to perform the operation whose power is $10.5 \mathrm{~W}$ for mediumpower setting. $\mathrm{O}_{2}$-plasma treatment can thoroughly clean the organic matter and simultaneously evacuate the pollutants by vacuum to avoid secondary pollution effectively. More importantly, this process will lead to the recombination among the chemical component on the surface of samples and form new surface features. Finally, a narrow $\mathrm{Au}$ electrode with $50 \mathrm{~nm}$ in thickness, which is suitable for $3 \omega$ measurement, was evaporated onto all the samples through a stainless steel mask by standard thermal evaporation method (insert of Fig. 2(b)). ${ }^{[18]}$ To eliminate sample-to-sample difference, all the electrodes of these samples were evaporated at the same time. This kind of four probe electrode will enable the accurate thermal resistance measurement crossing several interfaces.

\subsection{Interfacial thermal resistance measurement}

To obtain the interfacial thermal resistance of different samples, the differential $3 \omega$ method, which is one of the most common technique to measure the thermal conductivity of
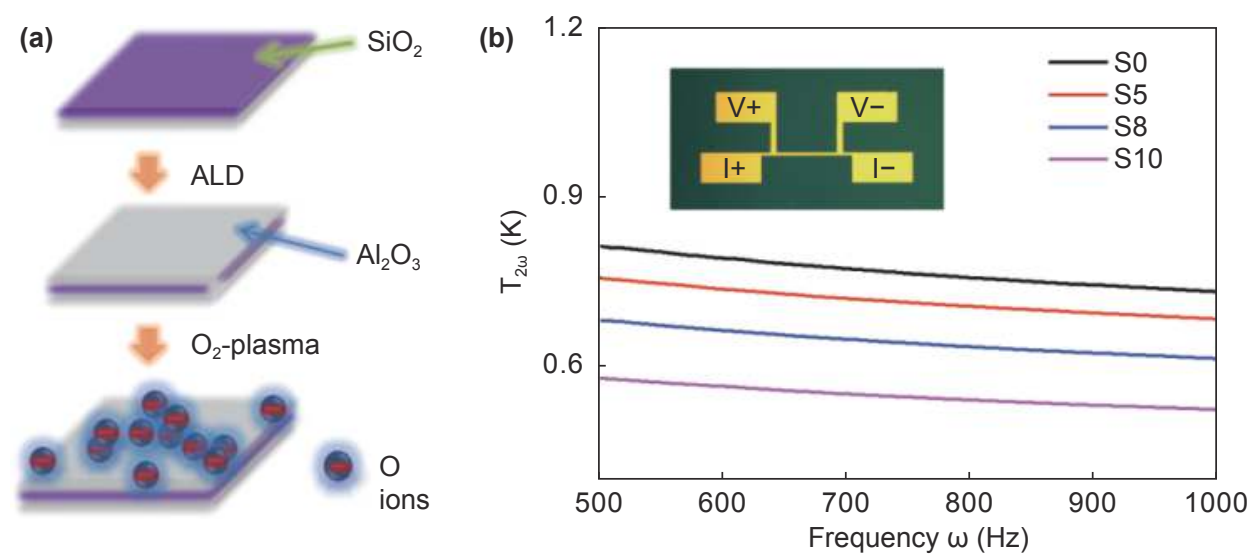

Fig. 2 (a) Fabrication process of metal-nonmetal interface. (b) Temperature changes versus the frequency of AC-current source for sample S0, S5, S8, S10. 
Table 1. The thermal resistance variation changes with the treatment time by $\mathrm{O}_{2}$-plasma at the room temperature.

\begin{tabular}{ccccccccc}
\hline Sample name & S0 & S4 & S5 & S6 & S7 & S8 & S9 & S10 \\
\hline Plasma time (mins) & 0 & 4 & 5 & 6 & 7 & 8 & 9 & 10 \\
$\Delta \mathbf{R}_{\text {th }}\left(\mathbf{1 0}^{-8} \mathbf{~ m}^{2} \mathbf{K} \mathbf{W}^{-1}\right)$ & $/$ & 1.40 & 2.06 & 6.41 & 9.24 & 8.10 & 9.94 & 13.52 \\
\hline
\end{tabular}

bulk material and thin film was employed in this work. ${ }^{[19]}$ For differential $3 \omega$ measurement, a thin metal electrode with specific shape and thickness is firstly deposited onto the sample. The $\mathrm{Au}$ electrode pattern is $50 \mu \mathrm{m}$ across and 1000 $\mu \mathrm{m}$ in length so that it can guarantee the one-dimensional heat flux from sample to substrate when a driven AC-current was applied. There is a linear relationship between its resistance and temperature, following $R=7.22+0.03561 T$ between the temperature range of $100 \mathrm{~K}-300 \mathrm{~K}$ in this experiment. Then a driven AC-current with varying frequency of $\omega$ was applied into this metal electrode so that this electrode could act as heater and temperature sensor simultaneously. A spontaneous temperature change with frequency of $2 \omega\left(T_{2 \omega}\right)$ will be obtained by detecting the voltage signal with frequency of $3 \omega$ $\left(V_{3 \omega}\right)$. Here, the temperature raise of electrode $T_{2 \omega}$ can be calculated from ${ }^{[20]}$

$$
T_{2 \omega}=2 \frac{d T}{d R} \frac{R}{V} V_{3 \omega},
$$

where $V_{3 \omega}$ is the detected $3 \omega$ voltage signal, $R$ is the resistance of electrode and $V$ is the voltage with frequency of $1 \omega$. This temperature raise will decrease with the raise of frequency, which is shown in Fig. 2(b). Then the differences in thermal resistance $\left(\Delta R_{\mathrm{th}}\right)$ between different samples can be obtained by

$$
\Delta R_{t \mathrm{~h}}=\frac{S \Delta T_{2 \omega}}{P},
$$

where $S$ is the cross-section area of sample below electrode, $P$ is the power of heat source, $\Delta T_{2 \omega}$ is temperature difference between samples. The thermal conductivity of silicon wafer from $T=100 \mathrm{~K}$ to $T=300 \mathrm{~K}$ can be detected simultaneously during the measurement of ITR. The thermal conductivity of S0 is about $\sim 113 \mathrm{Wm}^{-1} \mathrm{~K}^{-1}$ and $\sim 660 \mathrm{Wm}^{-1} \mathrm{~K}^{-1}$ at $T=300 \mathrm{~K}$ and $T=100 \mathrm{~K}$, respectively. It shows that $3 \omega$ measurement in this work is reliable by comparing to the reported result.

\section{Results and Discussions}

The ITR variations $\Delta R_{t h}$ of $\mathrm{Au}-\mathrm{Al}_{2} \mathrm{O}_{3}$ interface change as a function of the treatment time with $\mathrm{O}_{2}$-plasma at room temperature is shown in Fig. 3(a). The thermal resistance variations were obtained from $\Delta R_{t h}=R_{t h, 0}-R_{t h, i}(i=4,5,6,7$, $8,9,10$ mins), which is listed in Table 1 . With the increase of treatment time, the thermal resistances decrease dramatically, but their variations become more remarkably which vary from $\sim 1.40 \times 10^{-8} \quad \mathrm{~W}^{-1} \mathrm{~m}^{-1} \mathrm{~K}$ to $\sim 13.5 \times 10^{-8} \quad \mathrm{~W}^{-1} \mathrm{~m}^{-1} \mathrm{~K}$. We also measured the thermal resistance variation at different temperature (Fig. 3(b)). All the three samples show similar temperature dependence tendency that the thermal resistance variation is slightly increased with the improvement of temperature, which indicates that the treatment time with $\mathrm{O}_{2}$-plasma significantly change the thermal transport capability through metal- $\mathrm{Al}_{2} \mathrm{O}_{3}$ interface.

To demonstrate the essential reasons that influenced the ITR, we should ensure the factors that have been affected by $\mathrm{O}_{2}$-plasma. Firstly, treatment with $\mathrm{O}_{2}$-plasma may probably breakdown the $\mathrm{Al}_{2} \mathrm{O}_{3}$ film electrically, thus we measured the electrical resistance of $\mathrm{Al}_{2} \mathrm{O}_{3}$ after $\mathrm{O}_{2}$-plasma treatment for ten minutes (inset of Fig. 3(a)). This ultralow electrical conductivity indicates that $\mathrm{O}_{2}$-plasma did not breakdown the $\mathrm{Al}_{2} \mathrm{O}_{3}$ film and the electrical thermal conductivity of $\mathrm{Al}_{2} \mathrm{O}_{3}$ is negligible. Secondly, $\mathrm{O}_{2}$-plasma treatment with different time may lead to the variation of the surface roughness of $\mathrm{Al}_{2} \mathrm{O}_{3}$ layer. To verify whether the roughness is the factor that plays an important role in the interface thermal transport in this
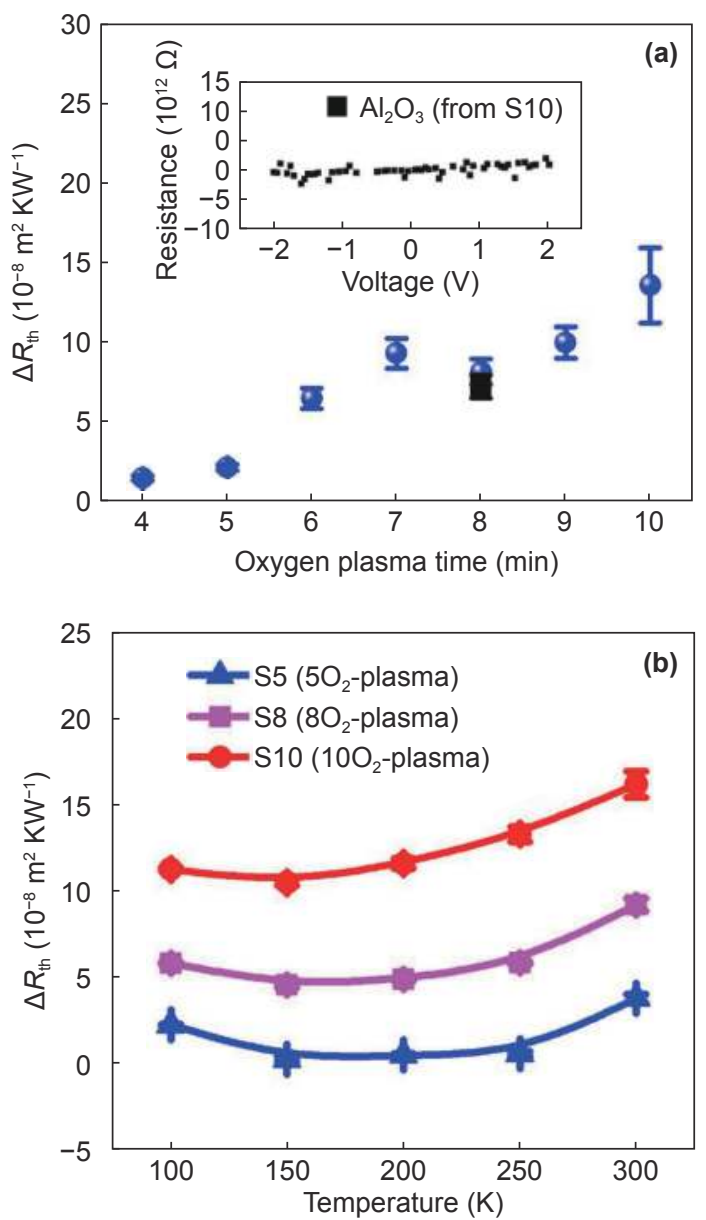

Fig. 3 (a) The thermal resistance variation changes as a function of the treatment time with $\mathrm{O}_{2}$-plasma under the room temperature $\Delta R_{t h}=$ $R_{t h, 0}-R_{t h, i}(i=4,5,6,7,8,9,10$ mins $)$. The black square is sample $\mathrm{S} 8$ ' which was kept in vacuum for two months after $\mathrm{O}_{2}$-plasma. Insert: electrical resistance of $\mathrm{Al}_{2} \mathrm{O}_{3}$ after plasma. (b) The thermal resistance changes versus the temperature. 
work, the AFM image for S0, S8, S10 are shown in Fig. 4. The roughness value of $\mathrm{Al}_{2} \mathrm{O}_{3}$ films keep within $0.3 \mathrm{~nm}-0.37 \mathrm{~nm}$, which provides specific evidence to support that the treatment of $\mathrm{O}_{2}$-plasma did not make obvious influence to the roughness of $\mathrm{Al}_{2} \mathrm{O}_{3}$ film. Thirdly, surface chemical bonds introduced by $\mathrm{O}_{2}$-plasma will also affect the ITR, ${ }^{[2]}$ which however will decay with time. To eliminate the effect the chemical bonds, we measured another sample (S8 '), whose electrode was evaporated two months (kept in a high vacuum chamber with vacuum level better than $1 \times 10^{-5} \mathrm{pa}$ ) after 8 -minutes $\mathrm{O}_{2}$-plasma (Fig. 3(a)). The result indicate that chemical bond could not explain the huge reduction in ITR. Finally, we focus on the carrier concentration, which could be obviously changed by the treatment of $\mathrm{O}_{2}$-plasma.

The $\mathrm{O}_{2}$-plasma treatment is believed to change the carrier concentration of $\mathrm{Al}_{2} \mathrm{O}_{3}$ on the surface and will open a new heat path: the electrons on metal transfer thermal energy to the electrons on surface state of nonmental, which will then transfer the energy to phonons of nonmetal through direct electron-phonon coupling (Fig. 1(b)) This new and strong electron-phonon coupling will open a new heat path between metal and nonmetal. ${ }^{[17]}$

To testify the contribution of electron-phonon coupling to ITR in this work, we measured the Hall resistance of graphene samples which were transferred onto the surfaces of different $\mathrm{O}_{2}$-plasma treated $\mathrm{Al}_{2} \mathrm{O}_{3}$ layers. The insert of Fig. 5(a) shows the schematic diagram for Hall measurement. The sample fabrication steps are same as Fig. 2(a). Subsequently, single layer graphene synthesized through chemical vapor deposition $(\mathrm{CVD})^{[19]}$ were transferred onto $\mathrm{Al}_{2} \mathrm{O}_{3}$ films (including $\mathrm{S} 0, \mathrm{~S} 5$, $\mathrm{S} 8, \mathrm{~S} 10){ }^{[2]}$ Then the standard E-beam lithography, thermal evaporation method and lift-off process were used to make suitable electrodes for hall measurement. We swept the magnetic field from $-5 \mathrm{~T}$ to $5 \mathrm{~T}$ at room temperature. The hall resistance $R_{x y}$ versus different magnetic field of $\mathrm{S} 0$ is plotted in Fig. 5(a). The carrier concentration of graphene samples onto different treated $\mathrm{Al}_{2} \mathrm{O}_{3}$ layers can be easily obtained by analyzing the slopes of hall resistance. According to the varied carrier concentration of graphene (Fig. 5(b)), it could be inferred that the carrier concentration of $\mathrm{Al}_{2} \mathrm{O}_{3}$ films have been changed by $\mathrm{O}_{2}$-plasma treatment. Note the measured carrier concentration of graphene is not the same with that in $\mathrm{Al}_{2} \mathrm{O}_{3}$, it only reflects the fact that carrier concentration on surface of $\mathrm{Al}_{2} \mathrm{O}_{3}$ changes. With the increase of $\mathrm{O}_{2}$-plasma treatment time, the carrier concentration of graphene is higher. So the higher carrier concentration of aluminum oxide will enhance the electron-phonon coupling through the metal-nonmetal interface (Fig. 1(b)), leading to the reduction of interfacial thermal resistance.

To further prove that $\mathrm{O}_{2}$-plasma treated devices has lower ITR and promising prospect on the application of heat dissipating devices, we reintroduce scanning thermal microscopy technique (SThM), a novel method, which could obtain the surface topography and temperature distribution simultaneously based on the atomic force microscopy. Fig. 6(a) shows the topographic image of a part of the graphene device, whose graphene sample was transferred onto sample S10. The non-treated graphene sample $\mathrm{S} 0$ is regarded as reference in this work. With the same DC-current applied onto the graphene films, this self-heating process will generate joule heating and lead to higher temperature near the center region (dashed square in Fig. 6(a)). Figs. 6(b-c) shows the temperature image
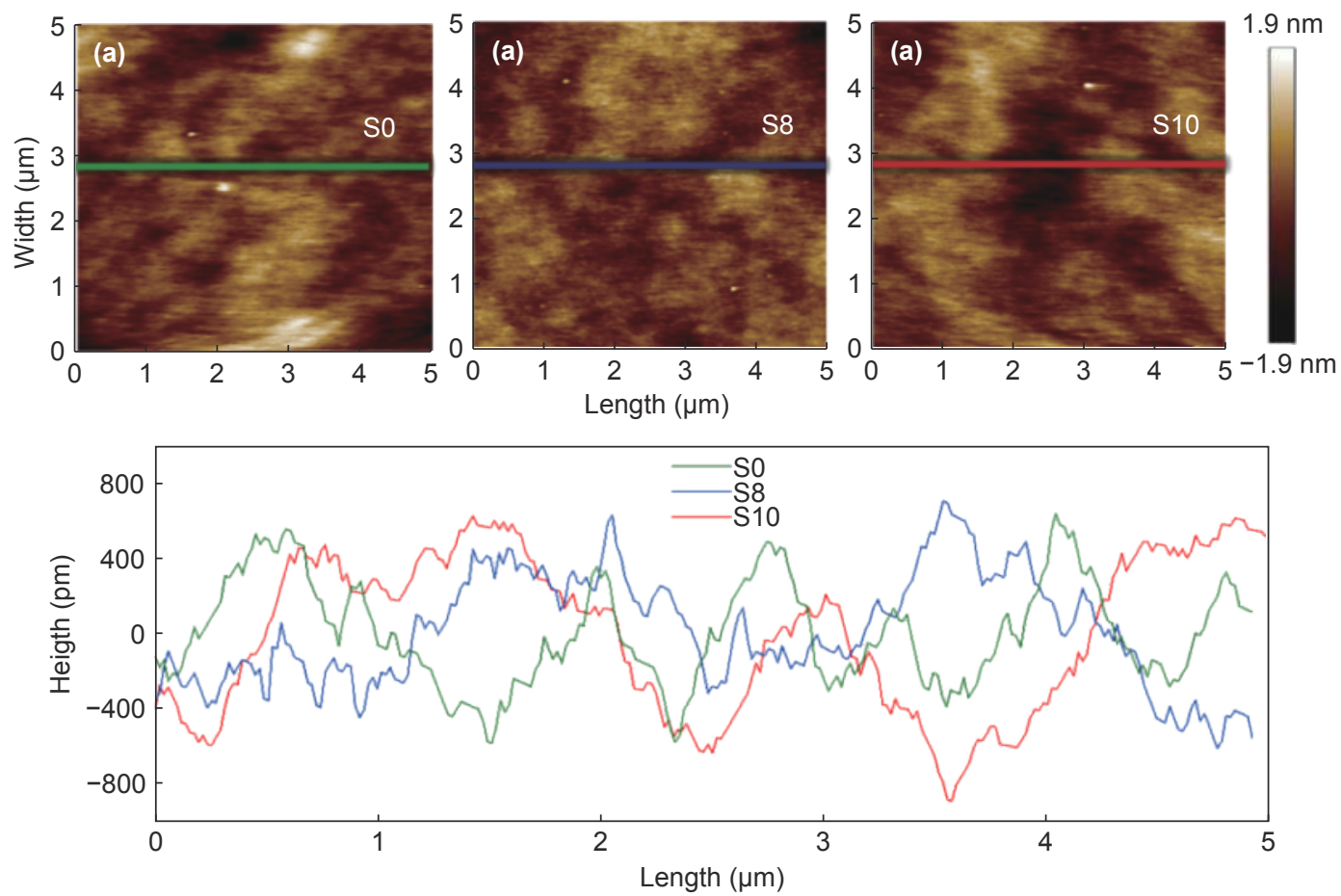

Fig. 4 The surface roughness of $\mathrm{Al}_{2} \mathrm{O}_{3}(\mathrm{~S} 0, \mathrm{~S} 8, \mathrm{~S} 10)$ measured by Atomic Force Microscope. 

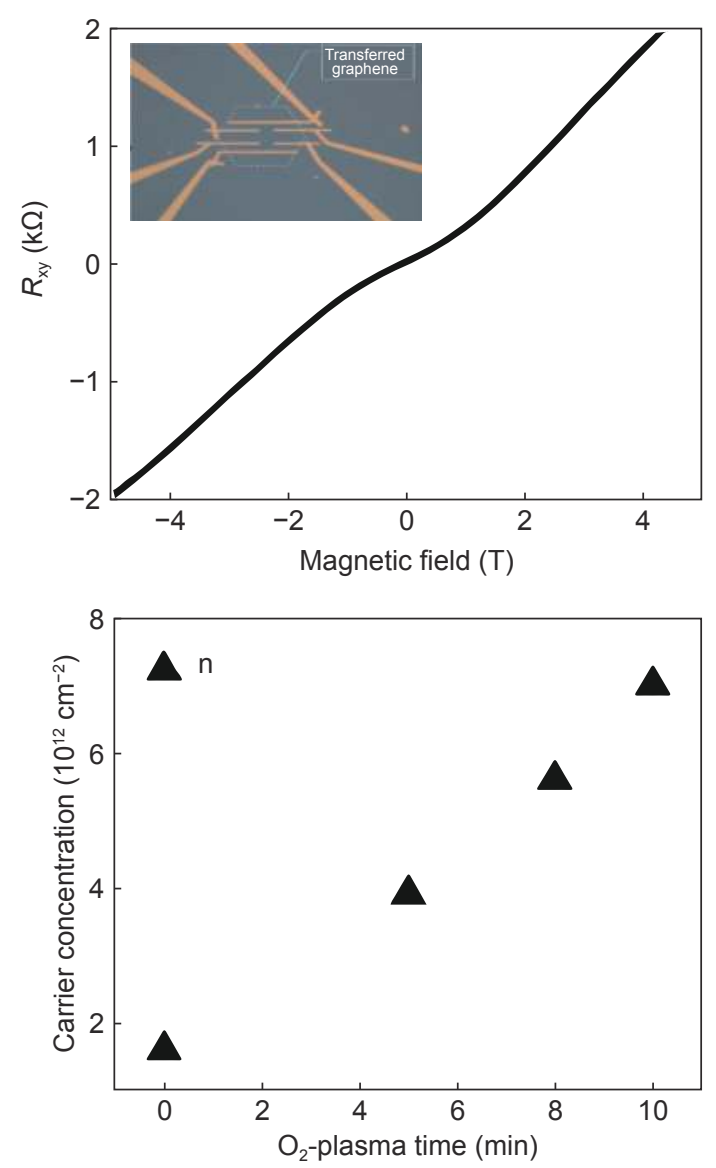

Fig. 5 (a) Hall resistance $R_{x y}$ versus magnetic field. The insert is the schematic diagram for hall measurement. (b) The carrier concentration of graphene under different $\mathrm{O}_{2}$-plasma treatment time.

of the graphene device S0 and S10 at the same high temperature region corresponded to power density of 1340 $\mathrm{W} / \mathrm{cm}^{2}$ and $2700 \mathrm{~W} / \mathrm{cm}^{2}$, respectively. The temperature variation of reference device is much higher than that of S10 (Fig. 6(e)), which directly illustrate that $\mathrm{O}_{2}$-plasma treatment effectively enhance the heat dissipation effect of metalnonmetal device.

\section{Conclusions}

In summary, we treated the surface of ALD-grown $\mathrm{Al}_{2} \mathrm{O}_{3}$ with $\mathrm{O}_{2}$-plasma with different time ranging from 4 to 10 minutes. We used differential $3 \omega$ method to measure the changes in the interfacial thermal resistance across $\mathrm{Au}-\mathrm{Al}_{2} \mathrm{O}_{3}$ interfaces. Results show that the change in interfacial thermal resistance increases with the plasma treatment time. The carrier concentration was extracted by measuring the Hall effect of graphene which was transferred onto the surface of $\mathrm{Al}_{2} \mathrm{O}_{3}$. We found a significant increase in the carrier concentration with the increase of plasma treatment time, which could induce a change in the relative Fermi level. Finally, we measured the temperature distribution of graphene- $\mathrm{Al}_{2} \mathrm{O}_{3}$ devices utilizing SThM technique, the better heat dissipation of $\mathrm{O}_{2}$-plasma treated sample indicating the promising prospect on the improvement of electronic devices.
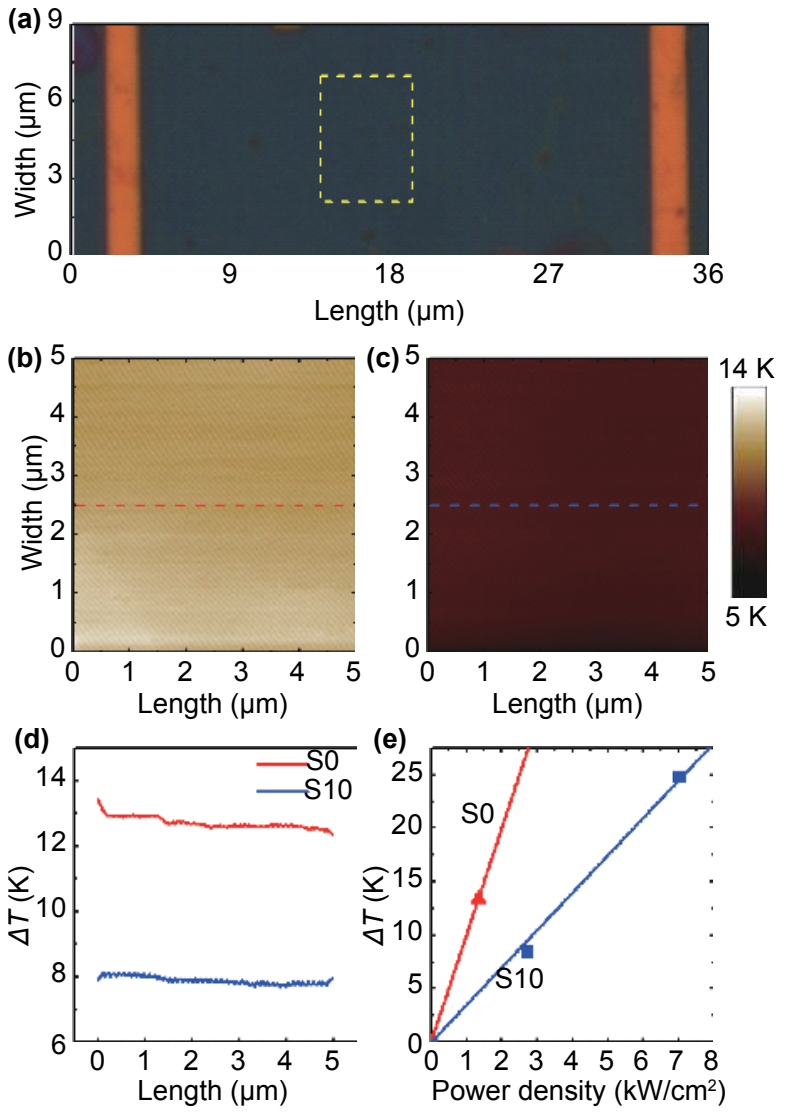

Fig. 6 AFM and SThM image of graphene device used. (a) AFM topological image of device, where bright parts are the electrodes. (b) Thermal image of graphene device (S0) heated at $I=5 \mathrm{~mA}$, represent square region in (a). (c) Thermal image of graphene device (S10) heated at $I=5 \mathrm{~mA}$, represent square region in (a). (d) The temperature profile of S0 and S10 of the dash line in (b) \& (c). (e) The relationship between temperature changes and power density. The slope of S0 is larger than S10, indicating better heat dissipation in S10.

\section{Acknowledgements}

This work was supported by the National Key R\&D Program of China (No. 2017YFB0406000), by the Key-Area Research and Development Program of Guangdong Province (No. 2020B010190004), by the National Natural Science Foundation of China (No. 11674245\& No. 11890703), and by the Shanghai Committee of Science and Technology in China (No. 17ZR1447900).

\section{Supporting information}

Not applicable

\section{Conflict of interest}

There are no conflicts to declare.

\section{References}

[1] D. Liu, X. Chen, Y. Yan, Z. Zhang, Z. Jin, K. Yi, C. Zhang, Y. Zheng, Y. Wang, J. Yang, X. Xu, J. Chen, Y. Lu, D. Wei, A. T. S. Wee and D. Wei, Nat. Commun., 2019, 10, 1188.

[2] W. A. Little, Can. J. Phys., 1959, 37, 334-349.

[3] D. A. Neeper and J. Dillinger, Phys. Rev., 1964, 135, A1028. 
[4] G. L. Pollack, Rev. Mod. Phys., 1969, 41, 48.

[5] E. T. Swartz and R. O. Pohl, Rev. Mod. Phys., 1989, 61, 605.

[6] D. G. Cahill, W. K. Ford, K. E. Goodson, G. D. Mahan, A. Majumdar, H. J. Maris, R. Merlin and S. R. Phillpot, J. Appl. Phys., 2003, 93, 793-818.

[7] M. Li, Y. Wang, J. Zhou, J. Ren and B. Li, Eur. Phys. J. B, 2015, 88, 149.

[8] I. M. Khalatnikov and I. N. Adamenko, J. Exp. Theor. Phys., 1973, 36, 391 .

[9] A. Majumdar and P. Reddy, Appl. Phys. Lett., 2004, 84, 4768-4770.

[10] M. I. Kaganov, L. E. M. and T. L. V., J. Exp. Theor. Phys., 1957, 4, 173-178.

[11] P. B. Allen, Phys. Rev. Lett., 1987, 59, 1460.

[12] W. A. Little, Phys. Rev., 1961, 123, 435.

[13] F. Wagner, F. J. Kollarits and M. Yaqub, Phys. Rev. Lett., 1974, 32, 1117-1120.

[14] S. V. Rotkin, V. Perebeinos, A. G. Petrov and P. Avouris, Nano Lett., 2009, 9, 1850-1855.

[15] K. H. Baloch, N. Voskanian, M. Bronsgeest and J. Cumings, Nat.
Nanotechnol., 2012, 7, 316-319.

[16] Y. K. Koh, A. S. Lyons, M. H. Bae, B. Huang, V. E. Dorgan, D. G. Cahill and E. Pop, Nano Lett., 2016, 16, 6014-6020.

[17] T. Lu, J. Zhou, T. Nakayama, R. Yang and B. Li, Phys. Rev. B, 2016, 93, 085433.

[18] D. J. Kim, D. S. Kim, S. Cho, S. W. Kim, S. H. Lee and J. C. Kim, Int. J. Thermophys., 2004, 25, 281-289.

[19] B. Liu, N. Xuan, K. Ba, X. Miao, M. Ji and Z. Sun, Carbon, 2017, 119, 350-354.

[20] D. G. Cahill, Rev. Sci. Instrum., 1990, 61, 802-808.

[21] H. Han, Y. Zhang, N. Wang, M. K. Samani, Y. Ni, Z. Y. Mijbil, M. Edwards, S. Xiong, K. Sääskilahti, M. Murugesan, Y. Fu, L. Ye, H. Sadeghi, S. Bailey, Y. A. Kosevich, C. J. Lambert, J. Liu and S. Volz, Nat. Commun., 2016, 7, 11281.

[22] G. Borin Barin, Y. Song, I. de Fátima Gimenez, A. G. Souza Filho, L. S. Barreto and J. Kong, Carbon, 2015, 84, 82-90.

Publisher's Note: Engineered Science Publisher remains neutral with regard to jurisdictional claims in published maps and institutional affiliations. 